\title{
QUAESTIONES INFORMATICAE
}

Vol. 1 No. 1

June, 1979

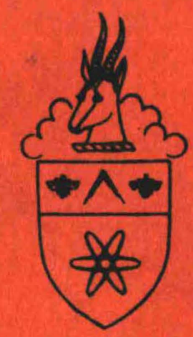




\title{
Quaestiones Informaticae
}

\section{An official publication of the Computer Society of South Africa 'n Amptelike tydskrif van die Rekenaarvereeniging van Suid-Afrika}

\author{
Editors: Dr. D. S. Henderson, \\ Vice Chancellor, Rhodes University, Grahamstown, 6140, South Africa. \\ Prof. M. H. Williams, \\ Department of Computer Science and Applied Maths, \\ Rhodes University, Grahamstown, 6140, South Africa.
}

\section{Editorial Advisory Board}

PROFESSOR D. W. BARRON

Department of Mathematics

The University

Southampton S09 5NH

England

PROFESSOR K. GREGGOR

Computer Centre

University of Port Elizabeth

Port Elizabeth 6001

South Africa

PROFESSOR K. MACGREGOR

Department of Computer Science

University of Cape Town

Private Bag

Rondebosch 7700

South Africa

PROFESSOR G. R. JOUBERT

Department of Computer Science

University of Natal

King George V Avenue

Durban 4001

South Africa

\section{Subscriptions}

Annual subscriptions are as follows:

$\begin{array}{llll} & \underline{\text { SA }} & \underline{\text { US }} & \underline{\text { UK }} \\ \text { Individuals } & \mathrm{R} 2 & \$ 3 & £ 1.50 \\ \text { Institutions } & \mathrm{R} 4 & \$ 6 & £ 3.00\end{array}$

MR. P. P. ROETS

NRIMS

CSIR

P.O. Box 395

PRETORIA 0001

South Africa

PROFESSOR B. VON SOLMS

Department of Computer Science

Rand Afrikaans University

Auckland Park

Johannesburg 2001

South Africa

PROFESSOR G. WIECHERS

Department of Computer Science

University of South Africa

P.O. Box 392

Pretoria 0001

South Africa

MR. P. C. PIROW

Graduate School of Business Administration, University of the Witwatersrand

P.O. Box 31170

Braamfontein 2017

South Africa 


\title{
Text Compression Techniques
}

\author{
J. E. Radue \\ Department of Computer Science, University of Natal, Durban.
}

\begin{abstract}
The benefits associated with text compression include more efficient use of peripheral devices, faster information transfer rates and, in some cases, improved sorting speeds through the use of shorter sort keys. However, these advantages must be balanced against a slight increase in CPU-time and the extra storage required for the associated code tables.

Information theory and statistics of English provide a background for the discussion of various text compression algorithms. The common objective of the methods described is to reduce the physical size of the text file while maintaining a complete representation of the information (reversible compression). The methods can be divided into two main classes:

(a) those that re-define the symbol codes to more accurate reflect the information content of each symbol, and

(b) those that use special codes to represent commonly occurring groups of symbols, thereby reducing redundancy due to mutual information between symbols.

Compression techniques not covered include those dealing with data files and with telemetry.

Finally, another approach to compression is described, which also holds some promise for automatic indexing and simpler inverted file design in document retrieval systems.
\end{abstract}

\section{Introduction}

Although the cost of both immediate access and secondary memory seems to be continually declining, it is still sound sense to use storage economically. Efficient storage, or compression, techniques currently implemented generally result in a reduction. by a factor of about 2 , in the storage required for a given amount of data. Put another way. using the same storage space, double the amount of data can be recorded. This is. however. not the hoped for panacea of the "need more disc-space" syndrome. as the compression algorithms do require some CPU time. and some data files may not be amenable to compression.

The common objective of the methods to be described here is to reduce the physical size of the text file while maintaining a complete representation of the information - termed reversible compression. Text compression. by which is meant reversible compression of documents or books written in a natural language, will be dealt with exclusively.

Other implications of compressing data, or text, include:

reduced data transmission costs

Much work is being done in the area of linking computers into networks, and the compression algorithms used include error detection and correction facilities. (These algorithms will not be dealt with here). However, even reducing the amount of useful information being transferred from secondary to main memory in one computer system could justify the use of compression.

\section{sorting}

Some forms of coding can achieve efficient compression while maintaining lexicographic ordering. The resultant shorter keys (and records) should speed up sorting, while data transmission time is also reduced.

distributable tapes

Computer-readable databases are currently distributed by abstracting and indexing services to act as the source for SDI services. If these are produced in compressed form, the encoding cost is incurred only once, while the subsequent cost of producing, and distributing. duplicate tapes is reduced through the use of smaller tapes. In the future, these databases will be distributed electronically within a network. where compression will still be advantageous.

\section{Background}

Any system that deals with natural language should take into account This paper was presented at the S.A. Computer Society Program 77 conference in Johannesburg. certain language characteristics. The properties of interest here are all obtained from studies in statistical linguistics, and are mainly formulae which have been found to describe experimentally obtained data.

The oldest vocabulary relation was popularised by Zipf [50], and has been the subject of many arguments and refinements (for example Mandelbrot [22] and Kucera and Francis [17]. "Zipf's Law" is as follows:

$$
p_{r}=0,1 / r
$$

where $p_{r}$ is the relative frequency (or probability) of a word of rank $r$ (Note: The types, or different words, in a sample of free text are ranked in order of decreasing frequency).

Despite criticisms, Zipf's Law is useful because of its simple form and because it is found to hold with useful accuracy for a variety of languages when sample sizes of the order of 100000 tokens are considered. Zipf's Law indicates that most tokens in a text consist of a small core of types, and in fact counts indicate that, for example, the 64 most frequent types in a sample of English text represents about $50 \%$ of the total tokens (Lesk [18].)

It has also been shown (Miller et al [25] and in Kucera and Francis [17] that if the relative frequencies of tokens of the same length are plotted against the logarithm of their respective length, a normal distribution is obtained. A relationship between the number of types and tokens in a large corpus has also been found experimentally (see Mandelbrot [23] and Schipma [34], and is as follows:

$$
\mathrm{D}=\mathrm{K} * \mathrm{~T}^{\mathrm{b}}
$$

where $\mathrm{D}$ is the number of word types, $\mathrm{T}$ is the number of tokens, and $\mathrm{K}$ and $\mathrm{b}$ are vocabulary dependent constants, 0 b 1 .

Information theory was developed by Shannon [42], and was soon applied to various fields of science. Shannon himself related information theory to linguistics (Shannon [43]. For a language in which characters occur independently, Shannon may be followed by defining the entropy, or the average amount of information per character in a large data set, as

$$
\mathrm{H}=\underset{-\mathrm{i}}{\boldsymbol{\Sigma}} \mathrm{p}_{\mathrm{i}} \log _{2} \mathrm{p}_{\mathrm{i}}
$$


where $\mathrm{p}_{\mathrm{i}}$ is the relative frequency, or probability, of the $\mathrm{i}$ th character and the summation is over the different characters in the character set.

When material is represented in binary code in the most efficient way, it can be shown (Shannon [43]) that this entropy will be the average number of binary digits required to encode each character. $\mathrm{H}$ is a symmetrical convex function with its maximum at the point

$$
\mathrm{p}_{1}=\mathrm{p}_{2}=\ldots=\mathrm{p}_{\mathrm{n}}=1 / \mathrm{n}
$$

In other words, the entropy function has a maximum value when all characters in the alphabet are equiprobable. This maximum value is $\log _{2} \mathrm{~N}$ bits, for an $\mathrm{N}$-character alphabet. Thus an efficient coding technique would assign 4,75 bits for a twenty-seven character alphabet.

This means that if a set of statistically independent messages is to be stored, the encoding which minimises storage space will be one that maximises the entropy, and that this occurs when the symbols of the encoding alphabet occur with as near as possible to equal probabilities. Huffman [14] has given a procedure for constructing such an optimum encoding, given a fixed set of messages (i.e.. character probabilities are fixed). This procedure will be discussed later.

The characters that occur in natural language text are neither independent (Burton and Licklider [4]) nor equiprobable (Pratt [29]). For example, some letters (in English. E. T. O, A. N, etc.), occur much more frequently than the rest of the alphabet. some letters tend to follow others. e.g. $\mathrm{U}$ after $\mathrm{Q}, \mathrm{H}$ after T. etc., and finally entire words or phrases tend to follow other words. Because these constraints extend over many letters (Burton and Licklider [4]), it is difficult to account for all of them. Shannon [43] estimates that the entropy for a large sample of English text ( 26 letters plus a blank), is about 1.4 bits per letter. That is, instead of the 4.75 bits of information which could be conveyed by 27 equiprobable. independent characters. English text only contains about 1,4 bits of information per character. This difference represents the redundancy of English. some of which can be eliminated by appropriate encoding schemes. Shannon [43] also showed that the entropy of English text varied with message length:

$\begin{array}{lcccc}\text { Message length (chars.) } & 1 & 2 & 3 & 1 \text { word + space } \\ \text { Entropy (bits/char.) } & 4,11 & 3,32 & 3,10 & 2,18\end{array}$

Further estimates, by other investigators, of the entropy of a word in English text vary from 1.7 to 2,0 bits/character (Schwartz and Kleiboemer [41].

The number of bits required to represent a character in a computer leads to a further difference. The most widely used character size is 8 bits ( 1 byte), which theoretically means that for each character in an English text corpus, 8 bits are being used to convey only 1,4 bits of information.

\section{Discussion of Algorithms}

From the discussion above, it can be seen that there are two basic principles involved in compression. Firstly, it may be possible to more accurately match the compression code to the information content of the input characters and secondly, the input characters may be clustered in some form (e.g., words bigrams or other fragments) to take advantage of the dependencies between characters. However, there is a vast "gray area" which consists of combinations of methods using both these general principles, and methods specially tailored to suit the peculiarities of the text or data being compressed. As a result, the following algorithms will be grouped under similarity of total method, rather than under one of the above two principles (Stoneburner [45]).

Although statistical techniques of compressing digital data will not be dealt with here, they are of fundamental importance in the transmission of data (e.g., from satellites). Typically these techniques include both compaction and error corection and are not reversible. The ad hoc techniques that depend only on the type of data being compressed, or on the file design, will also not be discussed (these latter techniques include datum subtraction or differences, abbreviations, etc.).

It must be kept in mind that thre are tradeoffs in the memory space required to store the code tables and in the time required to perform the appropriate transformation, and that some techniques are better suited to static files than to dynamic ones. It should also be noted that comparisons of the compression achieved by the various techniques are very difficult because of the different source files used.

\subsection{Fixed Length Coding for Character Strings}

\subsubsection{Character Repeat Suppression}

Although this technique works best on files which are known, in advance, to have long strings of repeating characters (such as program source files with strings of blanks), it is mentioned briefly here as the method is simple, execution is fast, memory requirements are small and appreciable compression can sometimes be achieved.

It consists of replacing a string of repeating characters with a code which describes the string composition. This replacement code is usually three characters long, the first being a unique, or special character (which is relatively rare in the file to be compressed), indicating the start of the code. The next two characters indicate, respectively, the character being suppressed and the repeat count (usually binary) of that character. Obviously only repeats of characters longer than the replacement code should be encoded by this method. If the special character is encountered in the data, it could be encoded as a repeat count of 1 .

A slight variation in the technique is used where it is known that many of the repeated strings consist of only a few characters, such as blanks or zeros. A different special character is used for each of these common repeated characters, resulting in a replacement code of only two characters - the indicator and the count.

\subsubsection{Bigram Substitution}

This technique makes use of the fact that, for some code sets (such as EBCDIC), the number of bit codes available is a great deal larger than the number of characters in the standard character set. The unused codes are substituted for the more frequently occurring bigrams. Theoretically, the maximum reduction achievable is $50 \%$, since, at best. one character substitutes for a bigram (Bookstein and Fouty [3]).

Various schemes have been devised and reported in the literature. Snyderman and Hunt [44] constrained their choice of encodable bigrams so that the initial characters belonged to a set of 8 "master" characters, selected primarily by frequency (see Pratt [29]), but arranged to include the vowels and the blank. The second characters belonged to a set of 21 "combining" characters, yielding a total of 168 encodable bigrams. All other characters were stored unaltered. Coding and decoding then depended in essence on the recognition of the "master" character.

Schieber and Thomas [33] placed no restrictions on the selection of bigrams (other than frequency), sacrificing the faster recognition of the previous scheme for an improved set of bigrams. A compaction of $43 \%$ was reported when 198 of the most common bigrams were encoded, compared to the $35 \%$ reported by Snyderman and Hunt [44].

Fouty [10] used bigram coding on databases of various languages and compared the performance to techniques based on variable-length coding (see 3.2). His results also indicate that it is possible to use a single set of bigrams for all the languages he considered (viz. English, German, French and Italian), and still achieve a compaction of about $40 \%$.

In general, bigram substitution appears to be well suited for use on active files of consistent provenance. The compression and decompression routines are relatively fast and economical of memory. The main drawbacks are the selection of the bigrams to be encoded. which is usually based on statistics obtained from a sample of the file, and the order in which the bigram substitutions are to be made. Note that this method can be combined with a fixed substitution for a small number of common character strings longer than two characters (preferably starting with one of the encodable bigrams, for ease of programming). 


\subsubsection{Common Phrase Suppression}

In this method a string of text is searched for repeating phrases (character strings) of any length. The phrases are then removed from the text and replaced by fixed-length reference numbers. The reference number is to an entry in a dictionary of phrases, contained in memory.

The two main problems associated with this method are firstly, the choice of a good set of phrases, and secondly, the use of the phrases in an order that will achieve best compression. These problems are illustrated in the following example. It should be noted that these two difficulties are common to many other compression techniques (see 2 above and 3.2 below), and various algorithms have been published to help minimise their effect (McCarthy [24], Wagner [47], Schuegraf and Heaps [36] and [37], Doucette [9], for example.)

Consider the input string "ABCXABCYABCZXABCY". If we choose the phrases "XABCY" and "ABC", and apply them in that order to the string, we get a compressed string of length 5 characters (assuming that the reference number is 1 character long). However, as "ABC" occurs 4 times, we could have chosen it first instead, thus obtaining a compressed string of 9 characters in length. Note that in general substituting for the longest phrase first does not necessarily give the best compression.

The overhead at compression time is relatively high as quite a bit of processing is needed both to find the optimal set of phrases to be suppressed (McCarthy), and to find the best substitution order (Schuegraf and Heaps [36] and [37] Doucette [9]. If the text is reasonably stable, however, the phrase set selection algorithm need only be run once. Storage savings of from $27 \%$ (Schuegraf and Heaps [37] to $58 \%$ (McCarthy [24]) have been reported, although the latter figure was obtained through combining this method with Huffman encoding of the phrase references and characters.

Note that the use of text and word fragments is very similar to this technique, but will be covered later because of their possible use as content indicators in automatic indexing.

\subsubsection{Adaptive Character String Substitution}

This is a more complex method than fixed substitution (3.1.3. above), with resultant higher overhead in time and space. The compression achieved is good and no preliminary generation of a code table is necessary. A brief description follows (Stoneburner).

The compressor starts with its code tables empty, except for one entry for each character in the input set. The input text is scanned and a count kept of the occurrence of each bigram. When a specific count reaches a threshold value, the compressor automatically defines a substitution code for that bigram. The definition is passed to the decoder as a special instruction in the compressed data. The process is iterative in the sense that counts are kept for the use of defined substitution codes in combination with other substitution codes or characters. Thus, although each substitution code is defined in terms of two other characters or substitution codes, it may represent a long string of characters in the original text. For example, a long string of X's in the input will result in the definition of a code for XX (say /), then the definition of a code for // (say $\$$, which represents $\mathrm{XXXX}$ in the input), then a code for $\$ \$$ (representing $\mathrm{XXXXXXXX}$ in the input), and so on. Obviously some large tables are needed and considerable time is spent searching and managing them. The decompressor only has to recognise a new substitution code definition and update its table accordingly. Decompression then consists of substituting the correct character string for each code in the compressed data.

On large files this technique results in a compression slightly better than a character-based Huffman code (Stoneburner). However, this depends on how "regular" the input stream is, and on how much of the file has been processed (due to "warm-up" or "learning"). It is thus suitable for files of several thousand words or more, and, due to the high overhead, inactive files are preferred.

\subsection{Variable length coding for Characters and Character Strings}

\subsubsection{Huffman Codes}

A procedure for producing a "minimum redundancy code" was described by Huffman [14], and minimises redundancy with respect to the element selected - normally the single character. All redundancy would be eliminated if the characters occured independently. Given this limitation, the procedure produces, for a finite set of characters with a given (fixed) frequency distribution, a set of codes with a minimum average length. In other words, the longest codeword is associated with the least probable character.

Additionally, when variable length codes are used, there must be a way to tell where one character ends and the next one starts. One of several ways of achieving this is to ensure that the code has the "prefix property", that is, no short code group is duplicated as the beginning of a longer group. Huffman codes have this prefix property.

The algorithm for generating a Huffman code is most easily illustrated by following an example. Assume a 7-character alphabet, with the following probability distribution:
A
0,3
B
0,15
$\mathrm{C}$
0.1
D
$\underset{0,25}{E}$
$\underset{0,04}{F}$
$G$
0,01

The characters are first ordered by probability. A coding tree is then built up by, at each stage, connecting the two lowest probabilities to a node which is assigned a value equal to their sum, and then placing this new node appropriately in the ranking. The process continues until only one node remains (see following figure):

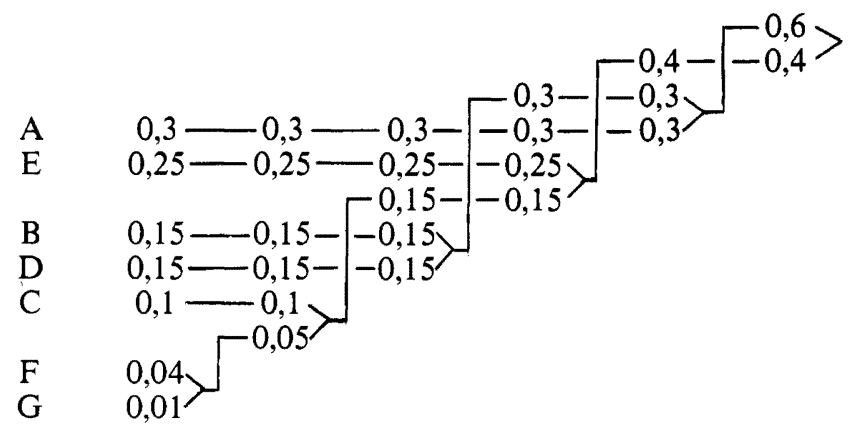

(Note that if the new node has a probability equal to another node in the list, the new node should be placed above the old node(s) which have equal probabilities. Schwartz [39] shows that this will minimise the codeword lengths).

The codes are then determined by assigning to each branch from a node a value of one or zero and reading from the root to each character.If, in the above figure, all upper branches are given a value " 0 " and all lower branches a value " 1 ", the following set of codes results:

$\begin{array}{lccccccc}\text { Character } & \text { A } & \text { E } & \text { B } & \text { C } & \text { D } & \text { F } & \text { G } \\ \text { Code } & 01 & 10 & 000 & 110 & 01 & 1110 & 1111 \\ \text { L }_{i} P_{i} & 0,6 & 0,5 & 0,45 & 0,3 & 0,45 & 0,16 & 0,04\end{array}$

For decoding purposes, it is convenient to have codewords of the same length adjacent to each other in the tree, which leads to the following code (Schwartz and Kallick [40]):
A
$\mathrm{E}$
01
B
100
$\mathrm{C}$
110
D
$\mathrm{F}$
G
$\begin{array}{lll}01 & 100 & 110\end{array}$
101
1110
1111 
Hoffman encoding works effectively on text and almost any other highly redundant data, usually achieving a compaction of about $50 \%$. It has also been frequently used on business-type data files. When combined with other methods such as blank suppression (Katcher [15]), slightly better results are obtained.

It is desirable that the statistical properties of the file being compressed do not change over a period of time. Thus a new code table may be needed for a file if the character frequencies have changed considerably. In fact, as can be appreciated, the most important single factor in the development of a Huffman code for a file is the choice of the base character set (for instance, see Ruth and Kreutzer [31]). If a Huffman code is based on a subset of the possible characters, a copy code should also be provided. This is a special codeword which is used to indicate that the character following it is reproduced exactly as it occured in the source file.

It should also be noted that it is possible to construct non-optimal prefix codes which satisfy arbitrary constraints and yet produce an average code lengtrh close to the optimum. One such constraint was used by Gilbert and Moore [11], in which the numerical value of the codes maintains the ordering of the input symbols (which may not be in probability order). This is useful in sorting coded alphabetical data. Gilbert and Moore give an example of text coding using their algorithm, and report an increase in cost of only 1,9\% over Huffman. Hu and Tucker [13] give an improved algorithm for the generation of such codes.

A practical disadvantage of all these minimum redundancy codes is that decoding must be done on a bit basis, usually by a time consuming tree search procedure.

\subsubsection{Other Methods}

In an attempt to speed up decoding and also to handle variable length codes on a fixed word length computer more efficiently, various other techniques have been proposed.

Bemer [2] assigned variable code lengths to words, and represented them by an integral number of bytes (and thus not optimum as in a Huffman code), the length of any code being indicated by the first bits of the code. This increased the coding speed by sacrificing some coding efficiency. A compression of $35 \%$ was achieved but a very large word dictionary had to be maintained. Heaps [12] gives a detailed description of a similar scheme, together with a thorough storage analysis, while particulars about the program design for coding and decoding are reported by Thiel and Heaps [46].

Mullarney [26] uses so-called optimal "self defining length codes". These are prefix codes with the additional property that the length of any code can be determined from an inspection of a fixed number $(\mathrm{k})$ of the initial bits of the code. Decoding is then reduced to two shifting and indexing operations per symbol: a shift of $\mathrm{k}$ bits, and their use to address a table; a further shift of the indicated number of bits, and their use to retrieve the decoded character from the table. Mullarney quotes an average cost increase of less than $5 \%$ over Huffman codes.

A consequence of the greater efficiency of any variable length encoding is increased sensitivity to bit errors, resulting in loss of synchronisation or incorrect deconcatenation of codes. Prefix codes are self synchronising, and Mullarney has shown that, with a deliberate perturbation of the coded data, synchronism is typically regained within 5-15 characters.

\subsection{Word Dictionary Techniques}

\subsubsection{Split Dictionary Encoding}

By dividing the word dictionary into several distinct sections, long words may be synthesised, instead of having a separate entry for each word to be encoded, as occurs in an integrated dictionary. This technique may be thought of as a sophisticated extension of the non-adaptive character string substitution methods already mentioned.

In a stem and suffix system, there are separate dictionaries for word stems and suffixes. The input string is scanned and whenever the stem of a compound word is found in the stem dictionary, the suffix dictionary is searched to see if it contains the suffix of the compound word. If it does, the word is replaced by codes for the stem and suffix (care must be taken of words with multiple suffixes).

Schwartz [38] shows that using a split dictionary of stems and suffixes allows more words to be encoded for a given dictionary size than could be encoded with an integrated dictionary. Schwartz included a table of common words thus also taking advantage of the more compact and faster encoding of an integrated dictionary. In a later paper (Schwartz and Kleiboemer [41], results show that for a text length of 19710 words, $79,49 \%$ were in the common word dictionary, $12,18 \%$ had to be synthesised and $8,33 \%$ were spelled.

Another similar method was described by White [49], in which frequent words and letter combinations (bigrams and trigrams) were held in a split dictionary. The encoder was designed to collect statistics of the input text and of the use of the dictionary, thus enabling some hand optimisation of the dictionary to be performed. The final dictionary contained 1340 entries and produced a claimed reduction of $47 \%$. Instead of using fixed length codes for the dictionary entries, it was calculated by White that Huffman codes would have produced a reduction of $4 \%$ more.

As these techniques do not have a dictionary entry for all possible words, a spelling mode is essential to enable words not listed to be spelled out character by character. If the dictionary can be fixed in advance, active files can be compressed by these methods.

\subsubsection{Intermediate Dictionary Compression}

This is basically a word dictionary technique and is due to Cullum [7]. It uses Huffman and run-length coding as integral parts of the method, and all words must appear in the dictionary. (The Huffman codes are defined algorithmically so that no code table is used).

The entire file is scanned to compile a complete dictionary of words and break characters (asterisk, period, comma, etc.). The break characters are then Huffman coded, based on their frequency count. while the words are "Huffman coded" assuming they are equiprobable (almost the same as assigning binary numbers to them), to save SPU time.

The file is then encoded as follows. A sub-string of say 1500 words and break characters is taken from the file. A binary "presence vector" (of length equal to the number of words in the dictionary) is then constructed by turning the corresponding bits on if the word that that bit represents is present in the sub-string. This presence vector defines the intermediate dictionary (ID), which consists of all the words corresponding to the bits set to 1 . The total number of words in the ID is counted and each word is assigned a "Huffman code" based on its position in the ID (see note on word coding above). The encoding for the string then consists of:

- The compressed presence vector in run-length encoded form

- The encoded word and break characters comprising the string in the order in which they appear. These are encoded by the concatenation of a 0 bit with the code for each break character or a 1 bit with the code for each word.

The main dictionary is included at the start of the compressed file and is followed by the codes for the break characters and then by the codes for all the sub-strings which together comprise the original file.

With the large overheads in this method it is suitable only for large inactive files. The files should also be subject to infrequent searches only, as searching must be done serially. Cullum reported a good compression of $53 \%$, recorded on a source file with many short words and misspellings (both of which are unfavourable to this method). 


\subsection{Binary Data Compression}

\subsubsection{Run-Length Encoding}

Some data tends to be in the form of sparse or low-density binary strings, that is, strings that have a preponderance of zero bits (or, similarly, of one bits). Such strings also arise in some of the compression techniques previously alluded to. A few of the many ways that have been devised to compress such strings will bo briefly mentioned here.

The count of zero bits between two consecutive one bits may be enconded using a Fibonacci code (Kautz [16]). These codes are variable length binary codes representing the positive integers and which have the pioperty that no code has a run of s consecutive ones, where $s$ is an integer dependent on the code and chosen by the user. The codewords are then separated by the insertion of strings of s ones (This method is useful only for strings with less than $10 \%$ ones.)

A1l alternative way to encode the counts of runs of zeros is by exponout-fraction encoding. Each integer is encoded as an $r$-digit exponent ( $r$ is fixed for the code) followed by a "fraction" having a number of bits equal to the binary value of the exponent. The fraction then gives the count of zeros.

In a method termed "asynchronous compaction". the following transforms are applied to the original binary string:
00
$0: 01--$
$11: 1-$
10

These reduce the number of zeros in the string, and are applied repeatedly until no further compaction results. Since the transform has a unique inverse. the original string can be reconstructed provided the number of times the transform was applied is known. (This could be supplied in a control field. along with the length of the compacted string). Long strings are compressed in sections. The method works well on strings that are not very sparse.

Lynch [19] biased the number of zeros in the source data by reassigning character codes ranked in order of the number of zero bits in a run to the characters ranked in order of frequency (thus the most frequent character is assigned the code with all zero bits). An extension of this technique assigns similarly ranked 2-byte codes to the bigrams ranked by frequency. Fixed 3-bit (and also 4-bit) codes are then used to represent runs of zeros (with one bits being encoded as runs of zeros of length zero). Reductions of the order of $30 \%$ were obtained using bigram biasing and 4 . bit codes.

\subsubsection{The COPAK Compressor}

The COPAK alphanumeric compressor (de Maine et al [8]) is a recursive bit pattern recognition technique. It is fully automatic and stores all control information necessary for decompression with the compressed data. The input can be any arbitrary string of characters. numbers, codes or bits. Compression is achieved with two basic bit pattern recognition routines (Type I and Type II) which operates in either slow- or fast-mode.

In Type I compression, a codeword is substituted for a recurring bit pattern in the data string to be compressed. (A codeword is a character which does not appear in the input.) In Type II compression. codewords are removed from the string, and their locations indicated by bit-maps. A bit-map is a bit string with one bit for each character position in the input string. Each bit that is turned on discloses a position in the data string where the particular codeword is to be inserted during decompression. For example. the string "computer science" could be represented as "c(1000000000100010)omputer siene". If we omit trailing zeros, and also use a bit map for e, the string becomes "c(100000000010001)$\mathrm{e}(0000010000101)$ omputr $\sin "$. In decoding, the substitution for e must precede that for $\mathrm{c}$. since the bit-map for c has positions for e's in the string.

The control information stored with the compressed data string contains the codewords, the bit patterns they replace, and the bit-maps for the codewords if used. Thus decoding is accomplished by stepping backwards through the control information of the string.
In the slow-mode of compression, the input string is searched to determine the most frequently recurring bit patterns to be replaced by codewords. If these bit patterns are supplied to thye COPAK compressor by the user, this step is eliminated, giving fast-mode compression.

Although this technique does not require much storage, the processing time is considerable, making it suitable for static files only. Its performance is comparable to adaptive character string substitution, achieving compressions of around $50 \%$ on natural language texts.

\subsection{Fragments}

\subsubsection{In Compression (See also 3.1.3)}

Walker [48] used variable length character strings (which he called Xgrams) to store names in a compressed form by concatenation of the codes for the X-grams. The set of X-grams, chosen on both frequency and length considerations, form a dictionary in which each entry is associated with a fixed length code.

Another proposal for the use of variable length character strings was made by Clare et al [5]. These string can be termed fragments since they are not limited to be elements of words, provided they are part of the record. (Word fragments on the other hand, refer to sub-strings of complete words only). Clare et al also placed another restriction on the choice of fragments, namely that they should occur with approximately equal frequencies in the file. The fragments are chosen such that they occur with a frequency below a certain threshold, and they are then used as the indexing, compression and retrieval elements.

Schuegraf [35] considers fragments such that there is no mutual overlap between the fragments, and which are chosen so that their frequency of occurrence is greater than or equal to a certain threshold (see also Schuegraf and Heaps [36] and [37], Doucette [9]. Schuegraf achieved a compression of about $60 \%$ on an issue of a MARC tape 51047 characters long.

Further implications and the construction of equifrequent character strings are discussed by Lynch [20]. The frequency of occurrence of characters and fragments is adjusted so that high frequency characters are "absorbed" into lower frequency fragments. The symbol set so obtained results in a higher entropy (because of near equal probabilities of occurrence) and can be used to generate, or encode, the source file.

\subsubsection{In Indexing and File Design}

In inverted file directories, fragments have been found to be reliable "surrogate words" (Schuegraf, Schipma [34] and they are also useful in file or bibliographic searhing (Colombo and Rush [6], Clare et al [5]. Lynch et al [21], Onderisin [28]. This is so particularly in files of consistent provenance: the fragments constitute the attributes which are employed singly, or in combination, to differentiate the items in the search file. (It has also been stated that fragments usually include or cover morphemes - Rickman and Gardner [30]. One could possibly consider fragments as computer equivalents of mnemonics, used for improving recoverability and discriminability (Norman and Bobrow [27]). Rickman and Gardner also showed that bigrams have a meaningful measure of association with index terms and can be used in automatic indexing. This was shown to hold for fragments as well by Barton et al [1] and Schuegraf

In addition, equifrequent fragments satisfy some of the conditions necessary for indexing terms to exhibit good discrimination (Salton and Wong [32].).

In inverted file design, the main advantages of using fragments instead of words is a reduction in the number of dictionary entries and the consequent saving of storage space. Schuegraf uses equifrequent fragments and, with the assumption that they are evenly distributed over all documents in the file. the inverted file directory consists of keys each with an approximately equal number of entries. Furthermore, the fragment list (or keys in the directory) is fixed even if the file increases in size (recall that the number of word types usually increases with an increase in the number of 
tokens). This leads to considerable simplification in the design of inverted files, allowing for easier updating.

\section{Conclusion}

Algorithms have been discussed which have been used by various investigators to compress text files. Although compression figures have been stated. it must be emphasised that they depend heavily on the type of text being compressed and on the number of bits used in the computer to represent a character. Until a model is presented which enables a theoretical evaluation of the relative effectiveness of the many compression techniques to be made, the technique used in a particular application will still usually be chosen by experience and experiment.
Fragments appear to hold great promise for both compression and automatic indexing. However. many aspects of their use still require investigation: for example. fragment distribution and selection to maximise their discrimination value: coding algorithms with indexing and/or compression as the main objective: and the determination of a fragment set over different files.

\section{Acknowledgement}

This work was carried out while the author was visiting the Depart ment of Computer Science at Cornell University, Ithaca. New York. I was partially supported by a bursary from the S.A. Council for Scientific and Industrial Research, which is gratefully acknowledged.

\section{References}

1. BARTON, I.J., CREASEY, S.E., LYNCH, M.F. and SNELL, M.J. (1974). An information-theoretic approach to text searching in direct access systems, Comm ACM, 17, 345-350.

2. BEMER, R.W. (1960). Do it by numbers - digital shorthand, Comm ACM, 3, 530-536.

3. BOOKSTEIN. A. and FOUTY, G. (1976). A mathematical model for estimating the effectiveness of bigram coding, Infor Proc and Man., 12, 111-116.

4. BURTON, N.G. and LICKLIDER, J.C.R. (1955). Long-range constraints in the statistical structure of printed English. The Amer J. of Psych., 68 650-653.
5. CLARE, A.C., COOK. E.M. and LYNCH, M.F. (1972). The identification of variable-length, equifrequent character strings in a natural language data-base, The Comp J., 15, 259-262.

6. COLOMBO, D.S. and RUSH, J.E (1969). Use of word fragments in computer based retrieval systems. J. of Chem Doc., 9. 47-50.

7. CULLUM, R.D. (1972). A method for the removal of redundancy in printed text. Ph. D dissertation. Dept. of Comp Sc., Univ. of Illinois.

8. DE MAINE. P.A.D., KLOSS. K. and MARRON, B.A. (1967). The SOLID System II. Part II Alphanumeric compression. U.S. 
Nat Bur of Standards, Tech Note 413.

9. DOUCETTE, V.L. (1977). An algorithm to select a fragment dictionary for data base compression, to be published in J. of the ASIS

10. FOUTY, G. (1973). Techniques for the compaction of machinereadable bibliographic records, MA dissertation, Grad Lib. School, Univ of Chicago.

11. GILBERT, E.N. and MOORE, E.F. (1959). Variable length binary encodings, Bell S!'stem Tech J., 38, 913-967.

12. HEAPS, H.S. (1972). Storage analysis of a compression coding for document data bases, Infor, 10, 47-61.

13. HU. T.C. and TUCKER, A.C. (1971). Optimal computer search trees and variable-length alphabetical codes, Siam J on Appl. Math., 21, 514-532.

14. HUFFMAN, D.A. (1952). A method for the construction of minimum-redundancy codes, Proc of the IRE, 40, 1098-1101.

15. KATCHER, A.M. (1971). Efficient utilization of limited access archival storage in a time shared environment, Proc of the Symp on Infor Stor and Retr, April 1-2, 1971, New York, 197-205.

16. KAUTZ, W.H. (1965), Fibonacci codes for synchronisation control, IEEE Trans on Info Theory, IT-11, 284-292.

17. KUCERA, H. and FRANCIS, W.N. (1967). Computational Analysis of Present-day American English, Brown Univ. Press. Rhodes Island.

18. LESK, M. (1970). Compressed text storage, Computer Sc Tech Report No. 3, Bell Tel Labs, Murray Hill, N.J.

19. LYNCH, M.F. (1973). Compression of bibliographic files using an adaptation of run-length encoding. Infor Stor and Retr., 9, 207214.

20. LYNCH, M.F. (1977). Variety generation - a reinterpretation of Shannon's mathematical theory of communication, and its impli cations for information science. J. of the ASIS, 28, 19-25.

21. LYNCH. M.F.. PETRIE, J.H. and SNELL, M.J. (1973). Analysis of the microstructure of titles in the INSPEC data-base. Infor Stor and Retr., 9, 331-337.

22. MANDELBROT, B. (1953). An informational theory of the statistical structure of language. In "Communication Theory", (Jackson, W., ed.) Academic Press, New York, 486-502.

23. MANDELBROT, B. (1961). On the theory of word frequencies and on related Markovian models of discourse, Proceedings 12th Symposium in Applied Mathematics, American Math Soc. 190219.

24. McCARTHY, J.P. (1974). Automatic file compression, in Int. Comp Symp, 1973. North Holland, Amsterdam, 511-516.

25. MILLER, G.A., NEWMAN, E.B. and FRIEDMAN, E.A. (1958). Length-frequency statistics for written English, Information and Control, 1, 370-389.

26. MULlaRNEY, A. (1975). Text compression, Ph.D. dissertation, Dept. of Comp Sc., Univ of Dublin.

27. NORMAN, D.A. and BOBROW, D.G. (1976). Recoverability and discriminability : factors in memory retrieval, Personal communication.

28. ONDERISIN, E.M. (1971). The least common bigram: A dictionary technique for computerized natural-language text searching, Proc. ACM Annual Conf., 82-96.
29. PRATT, F. (1939). Secret and Urgent : the Story of Codes and Ciphers, The Bobs Merril Co., New York.

30. RICKMAN, J.T. and GARDNER, H.W. (1973). On-line index term predictions using bigram-term associations, Proc. $A C M$ Annual Conf., 262-270.

31. RUTH, S.A. and KREUTZER, P.J. (1972). Data compression for large business files, Datamation, 18, 62-66.

32. SALTON, G. and WONG, A. (1976). On the role of words and phrases in automatic text analysis, Comp and the Humanities, 10, 69-87.

33. SCHIEBER, W.D. and THOMAS, G.W. (1971). An algorithm for compaction of alphanumeric data, J. of Lib Auto., 4, 198-206.

34. SCHIPMA, P.B. (1971). Term fragment analysis for inversion of large files, IIT Research Institute Research Report, 1-16.

35. SCHUEGRAF, E. J. (1974). The use of equifrequent fragments in retrospective retrieval systems, Ph.D. dissertation, Dept. of Computing Sc., Univ. of Alberta.

36. SCHUEGRAF, E.J. and HEAPS, H.S. (1973). Selection of equifrequent word fragments for information retrieval, Infor Stor and Retr., 9, 697-711.

37. SCHUEGRAF, E.J. and HEAPS, H.S. (1974). A comparison of algorithms for data base compression by use of fragments as language elements, Infor Stor and Retr., 10, 309-319.

38. SCHWARTZ,E.S.(1963). Adictionary forminimum redundancy coding, $J$. of the $A C M, 10,413-439$.

39. SCHWARTZ, E. (1964). An optimum encoding with minimum longest code and total number of digits, Infor and Control, 7, 3744.

40. SCHWARTZ, E.S. and KALLICK, B. (1964). Generating a canonical prefix encoding, Comm ACM, 7, 166-167.

41. SCHWARTZ, E.S. and KLEINBOEMER, A.J. (1967). A language element for compression coding. Infor and Control, 10, 315-333.

42. SHANNON. C.E. (1949). A mathematical theory of communication, in "The Mathematical Theory of Communication" by Shannon, C.E. and Weaver, W., The University of Illinois Press. Urbana. 3-91.

43. SHANNON, C.E. (1951). Prediction and entropy of printed English, Bell System Tech. J., 30, 50-64.

44. SNYDERMAN, M. and HUNT, B. (1970). The myriad virtues of text compression, Datamation, 16, 36-40.

45. STONEBURNER, P. (1977). Compression techniques, Personal communication.

46. THIEL. L.H. and HEAPS, H.S. (1972). Program design for retrospective searches on large data bases. Infor Stor and $R_{t} \prime l, ., 8$. $1-20$.

47. WAGNER, R.A. (1973). Common phrases and minimum-space text storage, Comm ACM, 16, 148152 and 183-185.

48. WALKER, V.R. (1969). Compaction of names by X-grams, Proc. of th Amer Soc for Inf Sc., 6, 129-135.

49. WHITE, H.E. (1967). Printed English compression by dictionary encoding, Proc IEEE, 55, 390-396.

50. ZIPF, G.K. (1949). Human Behaviour and the Principle of Least Effort, Addison-Wesley, Massachusetts. 


\section{Notes for Contributors}

The purpose of this Journal will be to publish original papers in any field of computing. Papers submitted may be research articles, review articles, exploratory articles or articles of general interest to readers of the Journal. The preferred languages of the Journal will be the congress languages of IFIP although papers in other languages will not be precluded.

Manuscripts should be in double-spaced typing on one side only of Henderson or Prof. M. H. Williams at

Rhodes University

Grahamstown 6140

South Africa

Form of manuscript

Manuscripts should be in double-space typing on one side only of sheets of A4 size with wide margins. The original ribbon copy of the typed manuscript should be submitted. Authors should write concisely.

The first page should include the article title (which should be brief), the author's name, and the affiliation and address. Each paper must be accompanied by a summary of less than 200 words which will be printed immediately below the title at the beginning of the paper, together with an appropriate key word list and a list of relevant Computing Review categories.

\section{Tables and figures}

Illustrations and tables should not be included in the text, although the author should indicate the desired location of each in the printed text. Tables should be typed on separate sheets and should be numbered consecutively and titled.

Illustrations should also be supplied on separate sheets, and each should be clearly identified on the back in pencil with the Author's name and figure number. Original line drawings (not photoprints) should be submitted and should include all relevant details. Drawings, etc., should be about twice the final size required and lettering must be clear and "open" and sufficiently large to permit the necessary reduction of size in block-making.

Where photographs are submitted, glossy bromide prints are required. If words or numbers are to appear on a photograph, two prints should be sent, the lettering being clearly indicated on one print only. Computer programs or output should be given on clear original printouts and preferably not on lined paper so that they can be reproduced photographically.

Figure legends should be typed on a separate sheet and placed at the end of the manuscript.

\section{Symbols}

Mathematical and other symbols may be either handwritten or typewritten. Greek letters and unusual symbols should be identified in the margin. Distinction should be made between capital and lower case letters between the letter $\mathrm{O}$ and zero; between the letter 1 , the number one and prime; between $\mathrm{K}$ and kappa.

\section{References}

References should be listed at the end of the manuscript in alphabetical order of author's name, and cited in the text by number in square brackets. Journal references should be arranged thus:

1. ASHCROFT, E. and MANNA, Z. (1972). The Translation of 'GOTO' Programs to 'WHILE' Programs, in Proceedings of IFIP Congress 71, North-Holland, Amsterdam, 250-255.

2. BÖHM, C. and JACOPINI, G. (1966). Flow Diagrams, Turing Machines and Languages with only Two Formation Rules, Comm. ACM, 9, 366-371.

3. GINSBURG, S. (1966). Mathematical Theory of Context-free Languages, McGraw Hill, New York.

\section{Proofs and reprints}

Galley proofs will be sent to the author to ensure that the papers have been correctly set up in type and not for the addition of new material or amendment of texts. Excessive alterations may have to be disallowed or the cost charged against the author. Corrected galley proofs, together with the original typescript, must be returned to the editor within three days to minimize the risk of the author's contribution having to be held over to a later issue.

Fifty reprints of each article will be supplied free of charge. Additional copies may be purchased on a reprint order form which will accompany the proofs.

Only original papers will be accepted, and copyright in published papers will be vested in the publisher.

\section{Letters}

A section of "Letters to the Editor" (each limited to about 500 words) will provide a forum for discussion of recent problems.

Hierdie notas is ook in Afrikaans verkrygbaar. 


\section{Quaestiones Informaticae}

\section{Contents/Inhoud}

A hardware-based real-time operating system $\ldots \ldots \ldots \ldots \ldots \ldots \ldots \ldots, 1$ M.G. Rodd

Real-time interactive multiprogramming 5

A.D. Heher

Distributed Computer Systems - a review

N.J. Peberdy

The P-NP question and recent independence results 26

N.C.K. Phillips

Text compression techniques

J.E. Radue 